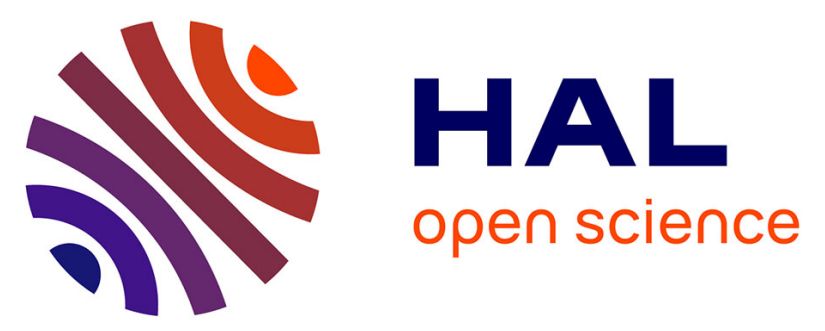

\title{
Correlated optical and structural analyses of individual GaAsP/GaP core--shell nanowires
}

Charlermchai Himwas, Stéphane Collin, Hung-Ling Chen, Gilles Patriarche, Fabrice Oehler, Laurent Travers, Omar Saket, François H Julien, J-C Harmand, Maria Tchernycheva

\section{To cite this version:}

Charlermchai Himwas, Stéphane Collin, Hung-Ling Chen, Gilles Patriarche, Fabrice Oehler, et al.. Correlated optical and structural analyses of individual GaAsP/GaP core-shell nanowires. Nanotechnology, 2019, 30 (30), pp.304001. 10.1088/1361-6528/ab1760 . hal-02349741

\section{HAL Id: hal-02349741 \\ https://hal.science/hal-02349741}

Submitted on 5 Nov 2019

HAL is a multi-disciplinary open access archive for the deposit and dissemination of scientific research documents, whether they are published or not. The documents may come from teaching and research institutions in France or abroad, or from public or private research centers.
L'archive ouverte pluridisciplinaire HAL, est destinée au dépôt et à la diffusion de documents scientifiques de niveau recherche, publiés ou non, émanant des établissements d'enseignement et de recherche français ou étrangers, des laboratoires publics ou privés. 


\title{
Correlated optical and structural analyses of individual GaAsP/GaP core-shell nanowires
}

\author{
C. Himwas ${ }^{1,2}$, S. Collin ${ }^{1}$, H.-L. Chen ${ }^{1}$, G. Patriarche ${ }^{1}$, F. Oehler ${ }^{1}$, L. Travers ${ }^{1}$, \\ O. Saket ${ }^{1}$, F. H. Julien ${ }^{1}$, J.-C. Harmand ${ }^{1}$, M. Tchernycheva ${ }^{1}$ \\ ${ }^{I}$ Centre de Nanosciences et de Nanotechnologies, UMR 9001 CNRS, Univ. Paris Sud, Univ. Paris- \\ Saclay, 10 Boulevard Thomas Gobert, 91120 Palaiseau Cedex, France \\ ${ }^{2}$ Semiconductor Device Research Laboratory, Department of Electrical Engineering, Faculty of \\ Engineering, Chulalongkorn University, 254 Phayathai Road, Bangkok 10330, Thailand
}

\begin{abstract}
We report on the structural and optical properties of $\mathrm{GaAs}_{0.7} \mathrm{P}_{0.3} / \mathrm{GaP}$ core-shell nanowires (NWs) for future photovoltaic applications. The NWs are grown by self-catalyzed molecular beam epitaxy. Scanning transmission electron microscopy (STEM) analyses demonstrate that the GaAsP NW core develops an inverse-tapered shape with a formation of an unintentional GaAsP shell having a lower P content. Without surface passivation, this unintentional shell produces no luminescence because of a strong surface recombination. However, passivation of the surface with a GaP shell leads to appearance of a secondary peak in the luminescence spectrum arising from this unintentional shell. The attribution of the luminescence peaks is confirmed by correlated cathodoluminescence and STEM analyses of the same NW.
\end{abstract}




\section{Introduction}

Semiconductor nanowires (NWs) have recently attracted attention as promising building blocks for future nanostructured devices due to several advantages. Thanks to the NW high aspect ratio, different III-V materials can be grown on foreign lattice-mismatched substrates or can be stacked within the NW without forming dislocations since the strain can be elastically relaxed by deforming the NW free surface. ${ }^{1} \mathrm{NW}$ arrays also possess advantageous optical properties. Namely, they have small optical reflectance and enhanced light diffusion, leading to an increased absorption in comparison to thin films. ${ }^{2,3}$ These attractive NW properties make them promising candidates for optoelectronic applications. Indeed, NWbased devices such as solar cells ${ }^{4-6}$, light emitters ${ }^{7-10}$, and photodetectors ${ }^{1-13}$ have been reported.

Despite reported advantages, one drawback of NWs for optoelectronic applications is their large surface-to-volume ratio leading to high surface losses. Surface states certainly act as non-radiative recombination centers for carriers and thus induce losses, which can be severe especially for the materials with high surface recombination velocity, like III-As ${ }^{14,15}$.

The impact of surface states can be reduced by depositing a passivating material on the NW surface. Different ex-situ passivations with chemical treatments (e.g. ammonium polysulfide solution ${ }^{16}$ or hydrazine-sulfide solution ${ }^{17}$ ) or by dielectric deposition (e.g. $\mathrm{PO}_{\mathrm{x}} / \mathrm{Al}_{2} \mathrm{O}_{3}$ shells ${ }^{18}$ ) have been studied. Alternatively, the surface can be passivated by epitaxially growing a shell of a wider band gap semiconductor directly after the NW synthesis (before exposing their surface to air). Compared to the post-growth treatments, the in-situ passivation does not introduce any contaminant at the interface with the passivating material and preserves the crystalline order of the core without any dangling bonds. For GaAs NWs, the most common in-situ passivation uses an AlGaAs shell. ${ }^{19-24}$ The choice of an alloy for this passivating material is usually made to preserve the direct band minima in the shell. Due to 
the proximity of the lattice constants between GaAs and AlAs $(\Delta \mathrm{a} / \mathrm{a}=0.14 \%)$, the shell can be grown rather thick before introducing a noticeable strain in the system. Songmuang et al. reported the optimal shell thickness of $\mathrm{Al}_{0.33} \mathrm{Ga}_{0.67} \mathrm{As}$ shell for $\mathrm{GaAs}$ core NWs to be $5-50$ $\mathrm{nm} \cdot{ }^{24}$ On the contrary, there are fewer reports on the shell passivation using III-P materials ${ }^{25-}$ ${ }^{28}$ and it is even more scarce to find a study of core-shell NWs when the core is a ternary alloy. Despite this lack of investigations, ternary alloy NWs and in particular GaAsP NWs ${ }^{26-28}$ are of great interest for photovoltaic (PV) applications. Indeed, the optimal band gap for a III$\mathrm{V}$ on $\mathrm{Si}$ tandem device lies around $1.7 \mathrm{eV}^{29}$ and therefore these devices require a ternary alloy for the top cell.

The selection of the GaAsP core material, which is well-suited for PV applications, introduces a lattice mismatch with AlGaAs, therefore the latter alloy is no longer a natural passivating material. In our previous work we found that a $5 \mathrm{~nm}$ thick $\mathrm{GaP}$ shell provides efficient passivation. This binary $\mathrm{GaP}$ shell was shown to yield a large enhancement of the luminescence intensity and carrier lifetime. ${ }^{28}$ In addition to the luminescence enhancement, passivation with this specific GaP shell was shown to result in a double emission peak in the GaAsP spectrum, which was at that time not fully understood. Such a spectral change is important for device applications and therefore it is crucial to understand its origin.

In this work, we focus on nanoscale analyses of the optical and structural properties of $\operatorname{GaAs}_{0.7} \mathrm{P}_{0.3} / \mathrm{GaP}$ core-shell NWs grown by molecular beam epitaxy (MBE). The origin of the double peak luminescence of the $\mathrm{GaAs}_{0.7} \mathrm{P}_{0.3} / \mathrm{GaP}$ core-shell NWs is clarified by performing correlated cathodoluminescence (CL) and scanning transmission electron microscopy (STEM) analyses on the same wire and by studying additional nanostructures (non-passivated GaAsP NWs) as a reference. The appearance of the double peak is attributed to the presence of an unintentional GaAsP shell having a lower P composition than the NW core, which only shows measureable luminescence when the NW surface is passivated with a GaP intentional 
shell. The compositional contrast between the core and the unintentional shell decreases with decreasing the growth temperature.

\section{Experimental Details}

Prior to the growth, $\mathrm{Si}(111)$ substrates were chemically etched by diluted HF 5\% for 2 min in order to completely remove the native oxide from the surfaces. A controlled $\mathrm{SiO}_{2}$ was then formed using a 1 min oxygen plasma treatment. This procedure was applied to obtain a reproducible $\mathrm{SiO}_{2}$ layer at the surface since it critically influences the nucleation of NWs. ${ }^{30}$ Subsequently, the substrates were outgassed in ultra-high vacuum (UHV) at $450{ }^{\circ} \mathrm{C}$ for 1 hour.

The growth of GaAsP NWs was carried out by solid-source MBE on intrinsic Si(111) substrates (Sil'tronix) at, if not mentioned otherwise, $600^{\circ} \mathrm{C}$ for $30 \mathrm{~min}$. The $\mathrm{NW}$ growth proceeded by the vapor-liquid-solid (VLS) mechanism catalyzed by Ga droplets, in the selfcatalyzed growth mode. ${ }^{31}$ The Ga flux was set equivalent to a two-dimensional deposition rate on GaAs (001) of $0.2 \mathrm{~nm} \cdot \mathrm{s}^{-1}$, as calibrated from reflection high-energy electron diffraction oscillations. Group V materials, As and $\mathrm{P}$, were supplied in the form of $\mathrm{As}_{4}$ and $\mathrm{P}_{2}$ by solidsource cells equipped with individual valves and shutters. The V/III beam equivalent pressure (BEP) ratios were set at $10-12$. The $\mathrm{P} /(\mathrm{P}+\mathrm{As})$ BEP ratio, defined as $\varepsilon_{\mathrm{P}}=$ $\mathrm{BEP}_{\mathrm{P} 2} /\left(\mathrm{BEP}_{\mathrm{P} 2}+\mathrm{BEP}_{\mathrm{As} 4}\right)$, was adjusted to 0.2 . Note that due to the different gauge sensitivities between different elements, the $\mathrm{P} /(\mathrm{P}+\mathrm{As}) \mathrm{BEP}$ ratio is not equal to the actual $\mathrm{P}$ concentration in the vapor phase. However, it varies proportionally with the actual P concentration.

For the core-shell structures, the growth proceeds in three steps: (i) NW core growth at $600^{\circ} \mathrm{C}$; (ii) Ga droplets crystallization; (iii) $\mathrm{GaP}$ shell radial overgrowth. The droplets crystallization step was simply performed by closing the Ga shutter (i.e. maintaining the same substrate temperature and group $\mathrm{V}$ fluxes as for the NW core growth). After the catalyst 
crystallization, the substrate temperature was decreased to $\sim 500^{\circ} \mathrm{C}$ prior to the shell growth step (iii). The absence of catalyst and the low substrate temperature favor the NW lateral expansion by vapor-solid (VS) growth. ${ }^{33,34}$

Then the GaP shell was deposited by opening the $\mathrm{Ga}$ and $\mathrm{P}$ shutters simultaneously. During the shell growth, the Ga flux and V/III BEP ratio were kept similar to those used for the NW growth. The shell deposition time was $2 \mathrm{~min}$, which results in a shell thickness of $5 \pm 1$ nm. According to our previous studies, ${ }^{28}$ this growth sequence yields the maximal enhancement of the $\mathrm{GaAs}_{0.7} \mathrm{P}_{0.3}$ core luminescence intensity.

The morphology of the NWs was first observed in a scanning electron microscope (SEM). Their structure was further analyzed by transmission electron microscopy (TEM) in a FEI Titan Themis microscope working at $200 \mathrm{kV}$. The TEM microscope is equipped with an aberration corrector on the probe to operate in STEM mode, and with a four-spectrometers Xray analyzer corresponding to 0.8 sr total angle of collection (Super X). For cross-sectional analyses, NWs were dispersed on a Si carrier substrate and were covered by a carbon film to fix their positions. Then they were cross-sectionally cut to obtain 80-100 nm-long NW segments using focused ion beam (FIB) technique. The optical characterization of the NW arrays was performed by photoluminescence (PL), while single dispersed NWs were characterized by CL. Steady state PL spectra were obtained at room temperature by exciting the NW ensemble with a continuous-wave $\operatorname{Ar}^{2+}$ laser $(\lambda=514 \mathrm{~nm})$ and collecting the emission with a Jobin Yvon HR460 monochromator equipped with a charge-coupled device (CCD) camera. Steady state CL measurements were performed at 10-20 K in an Attolight Chronos CL microscope, ${ }^{34}$ using an acceleration voltage of $6 \mathrm{kV}$ and an impinging current of $0.5-1 \mathrm{nA}$. The sample emission was collected by an achromatic reflective objective and dispersed by a Jobin Yvon iHR320 monochromator (grating: 150-grooves/mm) equipped with an Andor Newton CCD camera $(1024 \times 256$ pixels, pixel width $26 \mu \mathrm{m}$, dispersion per pixel: $0.53 \mathrm{~nm})$. 


\section{Results and discussion}

In our previous study on GaP passivated GaAsP NWs, we observed reproducibly an unintentional GaAsP shell with a lower $\mathrm{P}$ content just under the final GaP shell. ${ }^{28}$ To determine at which growth stage this unintentional shell is formed and how it affects the light emission from the NWs, we first characterize the structure and the luminescence of GaAsP NWs without the GaP passivating shell (section 3.1). Subsequently, we discuss the structural and optical properties of $\mathrm{GaAsP} / \mathrm{GaP}$ core-shell NWs, and the origin of the double luminescence peak in these structures (section 3.2).

\subsection{Structural and optical characterizations of GaAsP nanowires}

Figure 1(a) shows a TEM image of a GaAsP NW with a length of $\sim 3 \mu \mathrm{m}$ after the catalyst consumption. The obtained GaAsP NWs are mostly zinc-blende (ZB) phase except from the bottom and top regions. The NW bottom presents twinning over $\sim 200 \mathrm{~nm}$, while the NW tip, where the droplet crystallization took place, shows a characteristic sequence of different crystal phases, which was reported in the literature ${ }^{28}$ : starting with a pure ZB at the NW stem, the crystal first turns into a mixture of cubic and hexagonal phases, then to a pure wurtzite (WZ) phase and finally returns to a ZB phase at the topmost position. Detailed discussion about the crystal quality and crystal phase at different NW-heights can be found elsewhere. ${ }^{28}$

In figure 1(b), a cross-sectional high-angle annular dark-field (HAADF)-STEM micrograph of the GaAsP NW in «111 $\rangle$ zone axis is displayed. The HAADF brightness reveals four cross-sectional regions as indicated in the figure. HAADF brightness depends on the atomic number $(Z)$ of materials interacting with the beam and on the interaction volume. In our cross-sectional HAADF-STEM analysis, the interaction volume effect is minimized since the NW segment has a constant thickness in the [111] direction. We note that the cation $(\mathrm{Ga})$ was incorporated homogeneously in the NW cross-section so that the observed brightness modification in the cross-section can provide a qualitative compositional information of the 
element V. $Z_{\mathrm{As}}(33)$ is larger than $Z_{\mathrm{P}}(15)$, which implies that the second and the forth regions have an As-poor content while the As concentration increases for the first and the third regions.

Associated EDX maps for Ga and As elements performed in STEM mode are shown in figure 1(c). The EDX map for $\mathrm{P}$ element shows similar results with composition variation complementary to that of As element, hence we present here only the map for one anion (As). From the maps, Ga element is uniformly distributed over the NW cross-section while the measured As concentration changes in the four regions distinguished by the HAADF image (the changes are in agreement with the observed HAADF brightness modifications). The As concentration $\left(x_{\mathrm{As}}\right)$ deduced from EDX is 0.67 for the forth region, 0.71 for the first region, and 0.75 for the third region. For the second region where the HAADF contrast varies within less than a nanometer, we cannot precisely measure the concentration by EDX. However, the faint As element contrast suggests that the region has a comparatively lower As content. This attribution is supported by the dark HAADF contrast in the region which also suggests that the region is As-poor.

According to the growth mechanism, the first region was developed via step flow growth at the interface between the liquid droplet and the solid NW, creating prismatic GaAsP core. The ultra-thin P-rich second region was presumably created at the same time as the first region at the lateral surface of the NW core. The origin of the increased $\mathrm{P}$ concentration in region 2 remains to be explored. The third and the forth regions were grown around the previously-formed core by VS mechanism, creating GaAsP shell simultaneously with the axial NW elongation, which is referred to as "unintentional GaAsP shell". As suggested by Zhang et al., ${ }^{35,36}$ the prismatic shape of the previously-formed GaAsP core affects the As and $\mathrm{P}$ adatom diffusion on the facet driving As away from the edges, thus creating As-rich unintentional shell (third region) and P-rich corners (forth region). 
The cross-sectional observation allows analyzing only one region along the NW axis, which was preselected during the FIB cutting. To gain knowledge about the compositional variations along the wire axis, side-view STEM observations along the $\langle-211\rangle$ zone axis were performed. When the NW is observed from the side, the existence of the P-rich region 2 considerably affects the HAADF brightness yielding dark lines in HAADF micrograph at the core-unintentional shell boundary. Hence, the evolution of this boundary can be followed using conventional STEM in 〈-211〉 zone axis.

Figure 1(d)-(h) shows magnified HAADF-STEM micrographs in 〈-211〉 zone axis at various NW positions from the NW bottom to the NW top denoted in figure 1(a). These micrographs were processed to obtain clearer contrasts between the P-rich stripes (red arrows which are related to the core hexagonal peripheries) and the surrounding area. Despite the rather stable NW diameter equal to $85-89 \mathrm{~nm}$, the figures show that the GaAsP core diameter grown by VLS mechanism progressively increases from $43 \mathrm{~nm}$ to $89 \mathrm{~nm}$, and that the unintentional GaAsP shell grown by VS mechanism progressively decreases from $21 \mathrm{~nm}$ to 0 $\mathrm{nm}$. This unintentional shell thickness variation is indeed expected from a shell formed during the NW axial elongation. It is noteworthy that a simultaneous growth by VLS and VS mechanisms is not specific to the ternary alloy GaAsP NWs, this behavior was also observed for binary NWs (e.g. for GaP NWs). ${ }^{37}$

At the NW bottom region (i.e. in a $500 \mathrm{~nm}$-long segment from the NW-substrate interface) and at the NW top region (i.e. in a $500 \mathrm{~nm}$-long segment prior to the droplet consumption region), P-rich stripes related to the core hexagonal peripheries (region 2) were not observed in $\langle-211\rangle$ zone axis image. The disappearance of the stripes in the bottom region may be associated with a comparatively small diameter of the GaAsP core resulting in an insufficient HAADF contrast in the P-rich region 2. Indeed, Küpers et al. reported for a similar total NW diameter as in our case $(\sim 100 \mathrm{~nm})$, that the core diameter grown with VLS mechanism at the 
initial stage is only $25 \mathrm{~nm} .{ }^{38}$ Differently, the disappearance of the P-rich stripes in the top region is related to the absence of the unintentional GaAsP shell close to the NW top.

CL analyses of a GaAsP NW with a crystallized droplet were performed at low temperature (20 K). Figure 2(a) and (b) show the SEM image of an individual GaAsP NW and its corresponding CL intensity map integrated between wavelengths $500 \mathrm{~nm}$ and $900 \mathrm{~nm}$. Peak energy and peak full width at half maximum (FWHM) were extracted and plotted in figure 2(c) and (d). At the NW bottom (200 nm-long segment from the NW-substrate interface), the CL peak energy is located at $1.79 \mathrm{eV}$ associated with a broad linewidth of 120 meV. Then, it is abruptly blue-shifted to $1.83 \mathrm{eV}$ when moving upwards. When further moving to the NW top region, the CL peak energy is blue-shifted from $1.83 \mathrm{eV}$ to $1.85 \mathrm{eV}$, while the FWHM remains constant equal to $53 \mathrm{meV}$ over the NW length.

A comparatively low energy peak $(1.79 \mathrm{eV})$ at the NW bottom part may be associated with crystal phase heterostructures. Indeed, this region presents a high twin density as illustrated in STEM image of figure 2(e). For GaAs binary case, ${ }^{39}$ the phase heterostructure is reported to exhibit a type-II band alignment resulting in an emission peak red-shifted by up to $85 \mathrm{meV}$ from the $\mathrm{ZB}$ GaAs band gap. In our GaAsP alloy case, the luminescence from the crystal phase heterostrucutre is red-shifted by $40 \mathrm{meV}$ from the adjacent pure ZB GaAsP region (emitting at $1.83 \mathrm{eV}$ ). The increased broadening of the $1.79 \mathrm{eV}$ peak is also in favor of its attribution to crystal phase heterostructures.

In the NW stem, the peak energy extracted from the CL map was used to derive the composition $(x)$ by assuming that it follows a quadratic dependence, $\mathrm{E}_{\mathrm{g}}\left(\mathrm{GaAs}_{1-x} \mathrm{P}_{x}\right)=(1-$ $x) \cdot \mathrm{E}_{\mathrm{g}}(\mathrm{GaAs})+(x) \cdot \mathrm{E}_{\mathrm{g}} \cdot(\mathrm{GaP})-b x \cdot(1-x)$, reported for $\mathrm{ZB} \mathrm{GaAs} \mathrm{P}^{40,41}$ where $b$ is the bowing parameter taken equal to $0.19 .^{40}$ The derived $\mathrm{P}$ content at low temperature $(20 \mathrm{~K})$ is $0.27 \pm 0.01$, which is close to the value measured from the energy-dispersive $\mathrm{x}$-ray 
spectroscopy $(\mathrm{EDX})\left(\mathrm{cf}\right.$. figure $1(\mathrm{c}): x_{\mathrm{P}, \text { core }}=0.29$, and $x_{\mathrm{P} \text {, unintentional shell }}=0.25$ ). The observed emission can arise either from the NW core or from the unintentional GaAsP shell. However, if the CL peak was coming from the GaAsP unintentional shell, a strong modification of the CL emission along the NW axis should have been observed due to the variation of the shell thickness, which is not the case. Thus, we associate the CL peak with the emission of the GaAsP NW core. No optical signature of the unintentional GaAsP shell was observed in CL even though the measurement was carried out at low temperature, which is attributed to the surface non-radiative recombination. CL peak evolution from $1.83 \mathrm{eV}$ to $1.85 \mathrm{eV}$ when moving upwards along the wire axis, is associated with the tensile strain induced on the core by the unintentional GaAsP shell. This strain influences the CL by red-shifting the spectral response at the bottom region (to $1.83 \mathrm{eV}$ ) since the unintentional GaAsP shell here is comparatively thicker. The effect progressively decreases when moving towards the strainfree top region (where there is no unintentional GaAsP shell) with emission at $1.85 \mathrm{eV}$.

\subsection{Structural and optical characterizations of GaAsP/GaP core-shell nanowires}

To avoid wire-to-wire compositional variations inherent to spontaneously formed NW ensembles, correlated TEM-CL analyses were performed on the same NWs. The NWs were dispersed on a carbon-on-copper TEM grid to perform CL of a single NW at low temperature $(10-20 \mathrm{~K})$. The wire positions on the grid were recorded. After that, the structural characterizations were performed on the same nano-objects. This experimental sequence (the optical probe prior to the structural probe) was chosen to avoid any degradation of the NWs induced by the irradiation with high acceleration voltage electrons in STEM. However, in the manuscript we first report the structural results, since they are necessary for explaining the optical properties.

Figure 3(a) shows the HAADF-STEM image of one NW oriented along the $\langle 1-10\rangle$ zone axis. This NW has a length of $3.8 \mu \mathrm{m}$ and a diameter of around $120 \mathrm{~nm}$. Precise EDX 
measurements were performed in selected areas from the top, middle, and bottom regions (figures 3(b), (c), and (d), respectively) shown as white-dashed squares in figure 3(a).

The NW has a ZB structure with a twin density between 1 and 10 twin plane $\cdot \mu \mathrm{m}^{-1}$ except for the topmost part, where the structural sequence is identical to that of the GaAsP core, i.e. switching from a pure $\mathrm{ZB}$ phase to a highly twinned $\mathrm{ZB}$ phase, then to a $\mathrm{WZ}$ phase, and ending with a ZB phase at the topmost position.

The GaAsP core compositions ( $x_{\mathrm{P}, \text { core }}$ ) in the regions marked with red-dashed squares in figures 3(b), (c) and (d) were deduced from the EDX measurements. To extract them from the average core-shell compositions probed by EDX, we used a procedure described in the supporting information S1. The GaAsP core composition in the bottom region is $x_{\mathrm{P} \text {, core }}=0.21$ -0.24 (figure $3(\mathrm{~d})$ ) and it progressively increases to $x_{\mathrm{P}, \text { core }}=0.28-0.29$ in the middle region (figure 3(c)). At the NW-tip region (figure 3(b)), the core composition abruptly varies from its stable value of $x_{\mathrm{P} \text {, core }}=0.27$ (marked as " 4 ", "5") to $x_{\mathrm{P} \text {, core }}=0.21-0.23$ in the region grown during the catalyst crystallization (marked as "1", "2", and "3").

We note that defect loops were observed during the structural characterizations on the STEM images of the NW, that was previously analyzed by CL. Interestingly, the preferential sites for the defects are found around the twin boundaries as illustrated in bright-field (BF)STEM and in magnified BF-STEM images acquired in the middle region of the NW (figure 3(e), and (f)). Away from the twin planes, the defects also exist but with a lower density. At the bottom region of the NW where no twins were observed (figure 3(d)), such defects are evenly distributed. It is believed that the defects were generated due to electron irradiation during the $\mathrm{CL}$ experiment. These defects hindered the observations of the unintentional GaAsP shell. Therefore, we also performed the structural analyses of "fresh" NWs which were not subject to CL observations. 
Figure 4(a) depicts a HAADF-STEM image of a fresh NW in a $\langle-211\rangle$ zone axis. Highmagnification HAADF-STEM micrographs were collected at different positions marked as "b", "c", and "d", corresponding to the middle, close to the top, and the topmost position, respectively. In agreement with the structural results of the bare GaAsP core NW, the unintentional GaAsP shell disappears when moving upwards. The unintentional GaAsP shell thickness at the middle position is $5 \mathrm{~nm}$ as depicted in figure 4(b) while the existence of the unintentional GaAsP shell in figure 4(c) is ambiguous. If it exists, it should be thinner than 2 $\mathrm{nm}$ (the thickness progressively decreases from the middle position). At the NW topmost position (figure 4(d)), no unintentional shell was observed, but a modification of the GaAsP core during the droplets consumption process was evidenced. As seen from the diffuse contrast on the lateral surface, the GaAsP core in this region does not exhibit a well-defined hexagonal faceting but possesses rounded corners, surrounded with an obviously thicker $\mathrm{GaP}$ shell than in the GaAsP NW stem.

The CL spectra for the NW acquired at different positions are depicted from the bottom (spectrum\#1) to the top (spectrum\#56) of the NW in figure 5(a). According to the optical features, the spectra are grouped for discussion into 4 regions, namely region "1", "2", "3", and " 4 " as marked in the inset of figure 5(a).

In region " 1 ", CL shows a single luminescence peak which is located at $1.79 \mathrm{eV}$ at the NW bottom and globally blue-shifts to $1.82 \mathrm{eV}$ when moving upwards (blue-dashed line overlaid on the spectra \#1 to \#24 in figure 5(a)). The length of this region is $\sim 1.6 \mu \mathrm{m}$. Spectra from the bottom of the region where the correlated EDX measurements were performed (spectra \#1 to \#6), are magnified in figure 5(b). The luminescence spectra peaked at $1.79 \mathrm{eV}$ (with FHWM in the range of 35-40 meV) are attributed to the As-rich unintentional shell. The carriers created in the GaAsP core relax to the energy minimum in the As-rich unintentional shell before optical recombination, which explains the absence of the core luminescence. (The 
GaAsP core possesses a small diameter in the bottom part of the NW as discussed earlier.) The band gap energy $(1.79 \mathrm{eV})$ was calculated from the composition obtained from the EDX measurements at the bottom region (figure 3(d)), it is marked in figure 5(b) with a gray line. The derived band gap value is close to the experimental CL peaks. However, the EDX measurement englobes both the unintentional GaAsP shell and the NW core, so it can only give an indicative value. A slight peak blue-shift from spectrum \#1 to spectrum \#6 may be due to the strain induced by the GaP passivation shell, which has an increasing thickness due to shadowing effect (i.e. it is negligibly narrow at the bottom and becomes broader towards region “2”).

In region "2", the CL spectra show two luminescence peaks which are present over $\sim 1.4 \mu \mathrm{m}$ (marked with blue-dashed lines overlaid on the spectra \#25 to \#43 in figure 5(a)). The lower energy peak which continuously develops from the region "1", is associated with the As-rich unintentional shell while the higher energy peak is associated with the GaAsP core. We note that the core thickness in region " 2 " is higher compared to region " 1 ", which explains why its luminescence signature becomes visible contrary to the bottom NW part. Moreover, the large extension of the region " 2 " excludes the possibility that the two luminescence peaks are due to the carrier diffusion along the NW axis, but confirms that they originate from two distinct optically-active areas at the same point along the NW axis. The lower (higher) peak energy is $1.82 \mathrm{eV}(1.88 \mathrm{eV})$, which is blue-shifted to $1.84 \mathrm{eV}(1.89 \mathrm{eV})$ when moving upwards. The FWHM of the peaks is in the range of 30-45 meV. The observed blue-shift is attributed to the compressive strain from the $\mathrm{GaP}$ shell, which has an increasing thickness, however it could be also due to a compositional variation of the ternary alloy. To rule out the second hypothesis, binary $\mathrm{GaAs} / \mathrm{GaP}$ core-shell NWs were analyzed (see supporting information S2), showing a $20 \mathrm{meV}$ blue-shifted luminescence when moving upwards. For the GaAs/GaP NWs the core has a constant composition, therefore the 
hypothesis of the strain-induced shift is confirmed. CL spectra \#35 to \#40, corresponding to the region where the composition was measured by EDX, are displayed in figure 5(c). The composition extracted from the EDX measurements (figure 3(c)) accounting for the NW core and the unintentional shell is $\mathrm{GaAs}_{0.71} \mathrm{P}_{0.29}$. The calculated band gap $(1.86 \mathrm{eV})$ reported in figure 5(c) is in between the double luminescence peak derived from the P-rich GaAsP core and the As-rich unintentional shell. Since the exact diameters of the both optically-active areas are unknown, the EDX data cannot be further exploited. It is noteworthy, that the peak arising from the As-rich unintentional shell (lower peak energy) shows a stronger blue-shifted (about $10 \mathrm{meV}$ ) than that of the GaAsP core (less than $2 \mathrm{meV}$ ). This means that the strain exercised by the GaP shell mostly accommodates in the As-rich unintentional shell.

In region " 3 ", CL shows a single peak located at $1.89 \mathrm{eV}$, with a FHWM of $35 \mathrm{meV}$. Its energy continuously follows the peak attributed to the NW core in region " 2 ". By correlating with the structural information (cf. figure 4(c)), the luminescence peak is attributed to the $\mathrm{GaAs}_{0.73} \mathrm{P}_{0.27}$ core (the composition is extracted from the correlated EDX measurements (figure 3(b)). According to structural analyses, the unintentional GaAsP shell in region " 3 " becomes very narrow or absent, which explains the absence of its luminescence in the CL spectra. The length of the region " 3 " is around $0.7 \mu \mathrm{m}$ (black dashed line overlaid on the spectra \#44 to \#51 in figure 5(a). The stability of the peak energy also suggests that the region possesses a uniform alloy composition and a stable GaP shell thickness. Zoomed CL response in the region " 3 " is also depicted in figure 5(d) (spectra \#49 to \#51) where CL peaks are fitted by Gaussian curves. The intensity of the peaks monotonically decreases and disappears when moving out of region " 3 ”. The band gap of strain-free $Z B$ GaAs $0.73 \mathrm{P}_{0.27}$ core is estimated at $1.84 \mathrm{eV}$ (grey line showed in figure 5(d)). The observed $50 \mathrm{meV}$-blue-shift with respect to this value is associated with the strain induced by the passivating GaP shell.

In region " 4 ", CL peak shows an abrupt shift to $\sim 1.74 \mathrm{eV}$ with a FWHM of $\sim 30 \mathrm{meV}$. 
(CL spectra in the region fitted by red Gaussian curves are shown in figure 5(d)). By correlating with structural results (cf. figure 3(b)), this luminescence is associated with the WZ-GaAs ${ }_{0.78} \mathrm{P}_{0.22}$ segment strained by the GaP shell. Today the band gaps of the WZ-GaAs and WZ-GaP are not well established. By taking the exciton energies of WZ-GaAs equal to $1.474 \mathrm{eV}^{42}$ and WZ-GaP equal to $2.09 \mathrm{eV}^{43}$ the exciton energy for WZ-GaAs $\mathrm{s}_{0.78} \mathrm{P}_{0.22}$ can be tentatively estimated as $1.58 \mathrm{eV}$. Our experimental CL peak shows a blue-shift of $160 \mathrm{meV}$ with respect to this estimation. This may be due to a poor precision of the estimation arising from the uncertainty in the binary compound values, but also to a strong shell-induced strain due to a small core-to-total radius ratio ( 0.81 in this region to be compared with 0.93 in the NW stem). The $17 \mathrm{meV}$ blue-shift of the CL peak (from spectrum \#51 to \#55) is associated with a higher compressive strain, owing to the reduced core-to-total radius ratio when moving towards the NW tip.

The GaAsP/GaP core-shell NW schematic figure 5(e) was constructed based on the structural and optical results. The 4 regions are denoted in the figure. White dots overlaid on the schematic illustrate the evolution of optically-active segments along the NW. The emission in the region " 1 " comes from the As-rich unintentional shell, the emission in the region "2" comes from both the unintentional shell and the GaAsP core, the emission in the region "3" comes from the GaAsP core, and the emission in the region "4" comes from the WZ-GaAsP segment at the NW tip.

The signal-to-noise ratio (SNR) in the CL spectra of the correlated TEM-CL study was quite low because the NWs were dispersed on a carbon-on-copper TEM grid. To improve the SNR, we also performed additional CL measurements on NWs dispersed on Si substrates. Similar variation of the spectral features was found along the NW axis as reported in supporting Information S3. The optical properties of $\operatorname{GaAs}_{1-x} \mathrm{P}_{x} / \mathrm{GaP}$ core-shell NWs with other $\mathrm{P}$ concentrations (namely with $0<x_{\mathrm{P}}<0.35$ ) were also analyzed by CL. Their 
luminescence shows similar features with 4 characteristic regions, similar to the above described case (see supporting information S4).

Finally, we analyse the impact of the growth temperature on the emission energy of different regions in $\mathrm{GaAsP} / \mathrm{GaP}$ NWs aiming to reduce the compositional difference between the GaAsP core and the unintentional shell. Indeed, the radial alloy variation is undesirable for optoelectronic applications. The growth temperature is known to affect the composition of GaAsP NWs. ${ }^{44,45}$ We hence investigate the optical properties of GaAsP/GaP core-shell NWs synthesized with different GaAsP core growth temperatures, namely $T_{s, \text { core }}=600^{\circ} \mathrm{C}, 575^{\circ} \mathrm{C}$, and $550^{\circ} \mathrm{C}$. The rest of the growth conditions were identical to those of the sample used for the correlated TEM-CL study. All samples were probed by macro-PL at room temperature. The PL signal averages the emission of a large number of NWs $\left(10^{3}-10^{4}\right.$ wires accounting for a laser spot of $\sim 100 \mu \mathrm{m})$. First, the PL features of the sample $T_{s, \text { core }}=600^{\circ} \mathrm{C}$ are interpreted based on the previously-described CL results, then the evolution of the PL peaks with the GaAsP core growth temperature is analyzed.

Figure 6(a) depicts the integrated CL spectrum at $10 \mathrm{~K}$ of the correlated TEM-CL NW. The spectrum is fitted with three Gaussian curves with peak energies equal to $1.73 \mathrm{eV}, 1.81$ $\mathrm{eV}$ and $1.89 \mathrm{eV}$. As previously discussed, these peaks are associated with the strained WZGaAsP at the NW tip, the strained As-rich GaAsP unintentional shell, and the strained GaAsP core. The RT-PL spectrum of the corresponding NW ensemble is shown in figure $6(\mathrm{~b})$ together with the spectra from samples grown at lower core growth temperatures. All the PL spectra were fitted with three Gaussian curves. At $T_{s, \text { core }}=600^{\circ} \mathrm{C}$, the fitted Gaussian peaks are located at $1.66 \mathrm{eV}, 1.74 \mathrm{eV}$, and $1.82 \mathrm{eV}$ with the PL spectral shape identical to that of the integrated CL spectrum. They are expectedly red-shifted compared to the $10 \mathrm{~K} \mathrm{CL}$ results due to the band gap variation with temperature. Based on the observed similarity, the PL peaks are attributed to the same NW regions, i.e. the WZ-GaAsP at the NW tip, the GaAsP unintentional 
shell, and the GaAsP core. When the core growth temperature is decreased, the energy of the low-energy peak attributed to the WZ-GaAsP is stable, while its intensity decreases. The energy and the intensity of the middle peak attributed to the GaAsP unintentional shell are both stable. On the contrary, the high-energy peak attributed to the GaAsP core shows a monotonic red-shift from $1.82 \mathrm{eV}$ at $T_{s, \text { core }}=600^{\circ} \mathrm{C}$ to $1.77 \mathrm{eV}$ at $T_{s, \text { core }}=550^{\circ} \mathrm{C}$. This redshift evidences that the GaAsP core grown using VLS mechanism has a lower P content when growing at a lower substrate temperature while the composition of the GaAsP unintentional shell grown by a VS mechanism is not very sensitive to the growth temperature. The variation of the peak energies with the growth temperature are summarized in the inset of figure $6(\mathrm{~b})$. It was previously reported for the GaAsP NWs grown by metalorganic chemical vapour deposition, that the decrease of the substrate temperature alters the kinetically controlled PH3 decomposition $^{46,47}$ and minimizes the unintentional GaAsP shell ${ }^{44}$. In our case, the temperature decrease changes the incorporation via catalyst (VLS-grown), but does not significantly affect the VS growth. This effect can be used to reduce the compositional contrast between the core and the unintentional GaAsP shell. 


\section{Conclusion}

We have analyzed $\mathrm{GaAs}_{1-x} \mathrm{P}_{x} / \mathrm{GaP}$ core-shell NWs grown by MBE. The $\mathrm{P}$ content $(x)$ for the core was analyzed by EDX and varied from 0 to 0.35 . The structural investigation of GaAsP core NWs showed the appearance of a spontaneous low-P shell with a varied shell thickness along the growth axis. Despite the presence of the 2 compositional regions along the wire radius, only the $\mathrm{CL}$ emission from the GaAsP core was observed because the carriers in the unintentional GaAsP shell non-radiatively recombine at surface states. However, the luminescence from the GaAsP core and of the unintentional GaAsP shell were observed at different positions along the wire axis when the ternary alloy NW was overgrown with a GaP passivating shell. The observation of these optical features requires both the presence of different compositional regions in the core (driven by the different growth mechanisms), and the passivation of surface states. All the $\mathrm{GaAs}_{1-x} \mathrm{P}_{x} / \mathrm{GaP}$ core-shell NWs with $0<x_{\mathrm{P}}<0.35$ showed similar CL features, namely: (i) a low energy peak in the bottom part; (ii) a high energy peak in the top part; and (iii) a low energy peak at the NW tip. By performing a correlated STEM-CL study, these three features were respectively associated to: (i) the P-poor concentration unintentional GaAsP shell; (ii) the GaAsP core; and (iii) the short section WZGaAsP at the NW tip. Finally, lowering the growth temperature is proposed as a method for minimizing the compositional contrast between the core and the unintentional GaAsP shell.

Acknowledgements: This work has been partially financially supported by ANR project "Hetonan" (grant no. ANR-15-CE05-0009-05), by EU ERC project "NanoHarvest" (grant no. 639052), and by Ratchadapisek Somphot Fund for Postdoctoral Fellowship, Chulalongkorn University. The Attolight cathodoluminescence tool was funded by public grants supported by the Region Ile-de-France in the framework of C'Nano IdF (nanoscience competence center of Paris Region), by the European Union (FEDER 2007-2013), and by the LabEx GANEX (ANR-11-LABX-0014) and NanoSaclay (ANR-10-LABX-0035) as part of the "Investissements d'Avenir" program managed by the French National Research Agency (ANR). 


\section{References}

1. Glas, F. Critical dimensions for the plastic relaxation of strained axial heterostructures in free-standing nanowires. Phys. Rev. B 74, (2006).

2. Hu, Y., LaPierre, R. R., Li, M., Chen, K. \& He, J.-J. Optical characteristics of GaAs nanowire solar cells. J. Appl. Phys. 112, 104311 (2012).

3. Heiss, M. et al. III-V nanowire arrays: growth and light interaction. Nanotechnology 25, 014015 (2014).

4. Wallentin, J. et al. InP Nanowire Array Solar Cells Achieving 13.8\% Efficiency by Exceeding the Ray Optics Limit. Science 339, 1057-1060 (2013).

5. Åberg, I. et al. A GaAs Nanowire Array Solar Cell With 15.3 \#x0025; Efficiency at 1 Sun. IEEE J. Photovolt. 6, 185-190 (2016).

6. van Dam, D. et al. High-Efficiency Nanowire Solar Cells with Omnidirectionally Enhanced Absorption Due to Self-Aligned Indium-Tin-Oxide Mie Scatterers. ACS Nano 10, 11414-11419 (2016).

7. Scofield, A. C. et al. Composite axial/core-shell nanopillar light-emitting diodes at $1.3 \mu \mathrm{m}$. Appl. Phys. Lett. 101, 053111 (2012).

8. Minot, E. D. et al. Single Quantum Dot Nanowire LEDs. Nano Lett. 7, 367-371 (2007).

9. Ra, Y.-H., Navamathavan, R., Park, J.-H. \& Lee, C.-R. Coaxial InxGa1-xN/GaN Multiple Quantum Well Nanowire Arrays on $\mathrm{Si}(111)$ Substrate for High-Performance LightEmitting Diodes. Nano Lett. 13, 3506-3516 (2013).

10. Wang, Q. et al. Highly efficient, spectrally pure $340 \mathrm{~nm}$ ultraviolet emission from $\mathrm{Al} \times \mathrm{Ga}$ 1- x N nanowire based light emitting diodes. Nanotechnology 24, 345201 (2013).

11. Kuyanov, P., McNamee, S. A. \& LaPierre, R. R. GaAs quantum dots in a GaP nanowire photodetector. Nanotechnology 29, 124003 (2018).

12. LaPierre, R. R., Robson, M., Azizur-Rahman, K. M. \& Kuyanov, P. A review of III-V nanowire infrared photodetectors and sensors. J. Phys. Appl. Phys. 50, 123001 (2017).

13. Yang, Y.-K. et al. High performance photodetectors based on high quality InP nanowires. Chin. Phys. B 25, 118106 (2016). 
14. Lloyd-Hughes, J. et al. Influence of surface passivation on ultrafast carrier dynamics and terahertz radiation generation in GaAs. Appl. Phys. Lett. 89, 232102 (2006).

15. Joyce, H. J. et al. Electronic properties of GaAs, InAs and InP nanowires studied by terahertz spectroscopy. Nanotechnology 24, 214006 (2013).

16. Tajik, N., Chia, A. C. E. \& LaPierre, R. R. Improved conductivity and long-term stability of sulfur-passivated n-GaAs nanowires. Appl. Phys. Lett. 100, 203122 (2012).

17. Alekseev, P. A. et al. Nitride Surface Passivation of GaAs Nanowires: Impact on Surface State Density. Nano Lett. 15, 63-68 (2015).

18. Black, L. E. et al. Effective Surface Passivation of InP Nanowires by Atomic-LayerDeposited Al2O3 with POx Interlayer. Nano Lett. 17, 6287-6294 (2017).

19. Titova, L. V. et al. Temperature dependence of photoluminescence from single core-shell GaAs-AlGaAs nanowires. Appl. Phys. Lett. 89, 173126 (2006).

20. Perera, S. et al. Nearly intrinsic exciton lifetimes in single twin-free GaAs/AlGaAs coreshell nanowire heterostructures. Appl. Phys. Lett. 93, 053110 (2008).

21. Parkinson, P. et al. Carrier Lifetime and Mobility Enhancement in Nearly Defect-Free Core-Shell Nanowires Measured Using Time-Resolved Terahertz Spectroscopy. Nano Lett. 9, 3349-3353 (2009).

22. Tomioka, K., Kobayashi, Y., Motohisa, J., Hara, S. \& Fukui, T. Selective-area growth of vertically aligned GaAs and GaAs/AlGaAs core-shell nanowires on $\mathrm{Si}(111)$ substrate. Nanotechnology 20, 145302 (2009).

23. Hoang, T. B. et al. Observation of free exciton photoluminescence emission from single wurtzite GaAs nanowires. Appl. Phys. Lett. 94, 133105 (2009).

24. Songmuang, R. et al. Determination of the Optimal Shell Thickness for Self-Catalyzed GaAs/AlGaAs Core-Shell Nanowires on Silicon. Nano Lett. 16, 3426-3433 (2016).

25. Couto, O. D. D. et al. Effect of a GaAsP Shell on the Optical Properties of Self-Catalyzed GaAs Nanowires Grown on Silicon. Nano Lett. 12, 5269-5274 (2012).

26. Holm, J. V. et al. Surface-passivated GaAsP single-nanowire solar cells exceeding 10\% efficiency grown on silicon. Nat. Commun. 4, 1498 (2013). 
27. Zhang, Y. et al. Self-Catalyzed Ternary Core-Shell GaAsP Nanowire Arrays Grown on Patterned Si Substrates by Molecular Beam Epitaxy. Nano Lett. 14, 4542-4547 (2014).

28. Himwas, C. et al. In situ passivation of GaAsP nanowires. Nanotechnology 28, 495707 (2017).

29. LaPierre, R. R. Theoretical conversion efficiency of a two-junction III-V nanowire on Si solar cell. J. Appl. Phys. 110, 014310 (2011).

30. Matteini, F., Tütüncüoglu, G., Potts, H., Jabeen, F. \& Fontcuberta i Morral, A. Wetting of Ga on $\mathrm{SiO}_{x}$ and Its Impact on GaAs Nanowire Growth. Cryst. Growth Des. 15, 31053109 (2015).

31. Colombo, C., Spirkoska, D., Frimmer, M., Abstreiter, G. \& Fontcuberta i Morral, A. Gaassisted catalyst-free growth mechanism of GaAs nanowires by molecular beam epitaxy. Phys. Rev. B 77, (2008).

32. Morkötter, S. et al. Demonstration of Confined Electron Gas and Steep-Slope Behavior in Delta-Doped GaAs-AlGaAs Core-Shell Nanowire Transistors. Nano Lett. 15, 3295-3302 (2015).

33. Colombo, C., Heiß, M., Grätzel, M. \& Fontcuberta i Morral, A. Gallium arsenide p-i-n radial structures for photovoltaic applications. Appl. Phys. Lett. 94, 173108 (2009).

34. www.attolight.com.

35. Zhang, Y. et al. Polarity-Driven Quasi-3-Fold Composition Symmetry of Self-Catalyzed III-V-V Ternary Core-Shell Nanowires. Nano Lett. 15, 3128-3133 (2015).

36. Zhang, Y. et al. GaAsP nanowires and nanowire devices grown on silicon substrates. in (ed. Razeghi, M.) 101110X (2017). doi:10.1117/12.2250588

37. Oehler, F. et al. Measuring and Modeling the Growth Dynamics of Self-Catalyzed GaP Nanowire Arrays. Nano Lett. 18, 701-708 (2018).

38. Küpers, H. et al. Diameter evolution of selective area grown Ga-assisted GaAs nanowires. Nano Res. 11, 2885-2893 (2018).

39. Spirkoska, D. et al. Structural and optical properties of high quality zinc-blende/wurtzite GaAs nanowire heterostructures. Phys. Rev. B 80, (2009). 
40. Vurgaftman, I., Meyer, J. R. \& Ram-Mohan, L. R. Band parameters for III-V compound semiconductors and their alloys. J. Appl. Phys. 89, 5815-5875 (2001).

41. Nelson, R. J., Holonyak, N. \& Groves, W. O. Free-exciton transitions in the optical absorption spectra of Ga As 1 - x P x. Phys. Rev. B 13, 5415-5419 (1976).

42. Novikov, B. V. et al. Photoluminescence properties of GaAs nanowire ensembles with zincblende and wurtzite crystal structure. Phys. Status Solidi RRL - Rapid Res. Lett. 4, $175-177$ (2010).

43. Assali, S. et al. Direct Band Gap Wurtzite Gallium Phosphide Nanowires. Nano Lett. 13, $1559-1563$ (2013).

44. Sun, W. et al. Spontaneous formation of core-shell GaAsP nanowires and their enhanced electrical conductivity. J. Mater. Chem. C 3, 1745-1750 (2015).

45. Metaferia, W. et al. GaAsP Nanowires Grown by Aerotaxy. Nano Lett. 16, 5701-5707 (2016).

46. Smeets, E. T. J. M. Solid composition of GaAs1-xPx grown by organometallic vapour phase epitaxy. J. Cryst. Growth 82, 385-395 (1987).

47. Larsen, C. A., Buchan, N. I. \& Stringfellow, G. B. Mass spectrometric studies of phosphine pyrolysis and OMVPE growth of InP. J. Cryst. Growth 85, 148-153 (1987). 


\section{Figure Captions}

Figure 1. (a) Bright-field TEM micrograph of a GaAsP NW after droplet crystallization in <211 > zone axis. (b) Cross-sectional HAADF-STEM micrograph of a GaAsP NW in 〈111〉 zone axis with (c) its associated EDX maps performed in STEM mode, showing Ga and As elements. (d)-(h) Magnified HAADF-STEM micrographs in 〈-211〉 zone axis of a crystallizeddroplet GaAsP NW, taken at various NW heights as marked in (a). Delimited image processing areas overlaid on the HAADF-STEM micrographs ((d)-(h)), show clearer contrasts of stripes (red arrows) and surrounding areas. Scale bars in (b)-(h) correspond to $50 \mathrm{~nm}$.

Figure 2. (a) SEM image of a single GaAsP NW. CL was performed on this NW, using the acceleration voltage of $6 \mathrm{kV}$ and the impinging current of $1 \mathrm{nA}$, at $20 \mathrm{~K}$. From the recorded spectral map, the integrated CL intensity (b), the peak energy (c), and the FWHM (d) maps were extracted. (e) High magnification TEM micrograph of the NW bottom, illustrating a high twin density region.

Figure 3. (a) HAADF-STEM image of the correlated GaAsP/GaP core-shell NW. High magnification HAADF-STEM micrographs collected at the NW tip (b), the NW middle (c), and the NW bottom (d). Red square delimited areas in panels (b), (c), and (d) were selected to performed EDX maps. The GaAsP core compositions for each selected area are shown. (e) Bright-field STEM and (f) Magnified bright-field STEM micrographs of the correlated $\mathrm{GaAsP} / \mathrm{GaP}$ core-shell NW, showing defect loops occurred in the NW.

Figure 4. (a) HAADF-STEM image of a fresh GaAsP/GaP core-shell NW. Zoomed HAADFSTEM micrographs performed at the (b) NW middle, (c) close to the top of the NW, and (d) NW tip, demonstrating the evolution of the unintentional GaAsP shell, and of the intentional GaP shell.

Figure 5. (a) $10 \mathrm{~K} \mathrm{CL}$ spectra for the correlated TEM-CL GaAsP/GaP core-shell NW obtained for different excitation positions from the bottom (spectrum \#1) to the top (spectrum \#56) of the NW. The inset shows a corresponding TEM image of the NW. (b) Zoomed $10 \mathrm{~K}$ CL spectra from panel (a) at the bottom of the NW (spectra \#01 to \#06). (c) Zoomed $10 \mathrm{~K} \mathrm{CL}$ spectra from panel (a) at the middle of the NW (spectra \#35 to \#40). (d) Zoomed $10 \mathrm{~K} \mathrm{CL}$ spectra from panel (a) at the tip of the NW (spectra \#49 to \#55).

Figure 6. (a) $10 \mathrm{~K}$ integrated CL spectrum of the correlated TEM-CL NW fitted with three Gaussian curves (red, black, and blue lines) superimposed on the experimental spectrum. (b). RT-PL spectra of $\mathrm{GaAsP} / \mathrm{GaP}$ core-shell NW ensembles grown at various core growth temperatures, namely $T_{s, \text { core }}=600^{\circ} \mathrm{C}, 575^{\circ} \mathrm{C}$, and $550^{\circ} \mathrm{C}$. Each spectrum is fitted with three Gaussian curves (red, black, and blue lines) superimposed on the experimental spectrum. The inset shows the variation of the peak emission energy as a function of the GaAsP core growth temperature. 
Figure 1

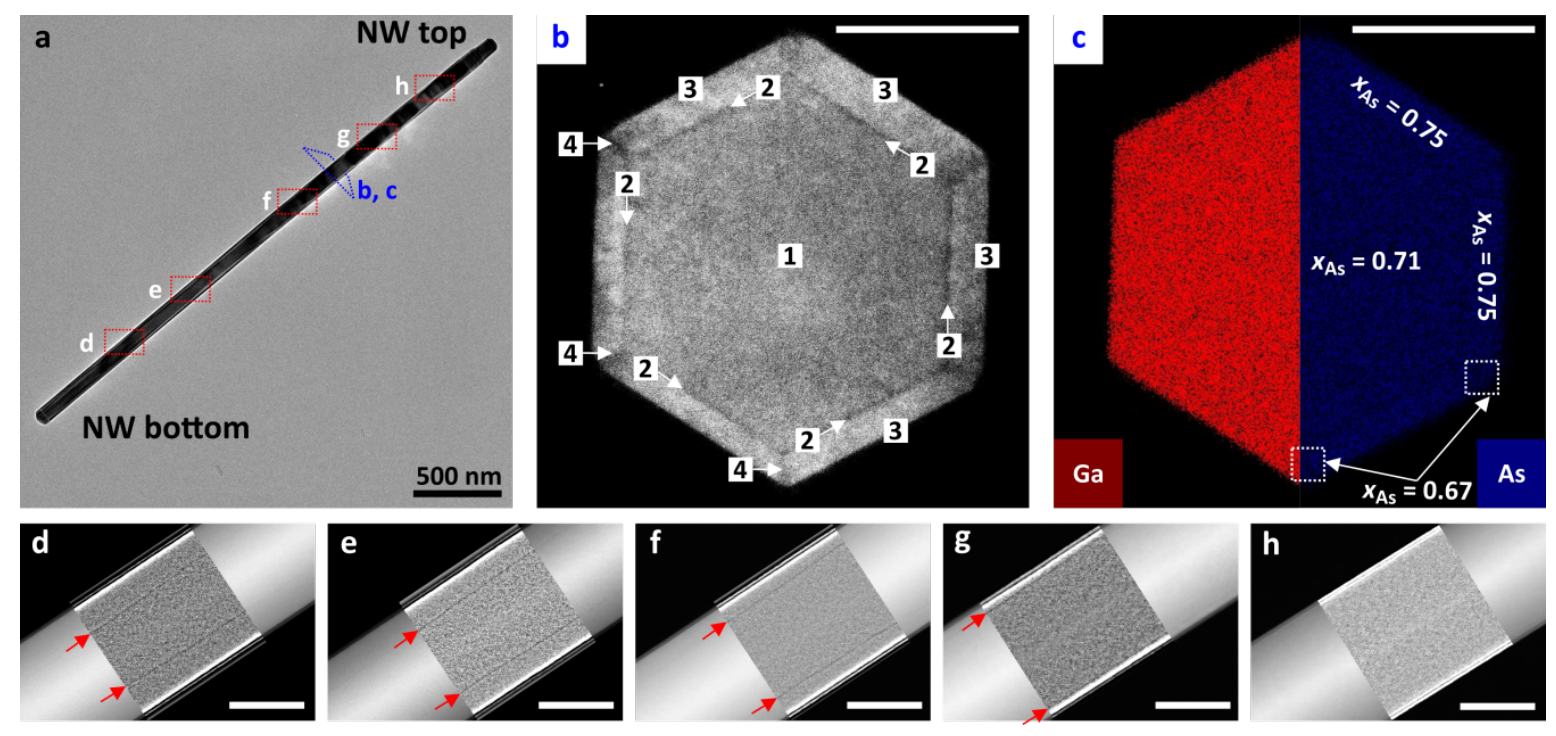


Figure 2
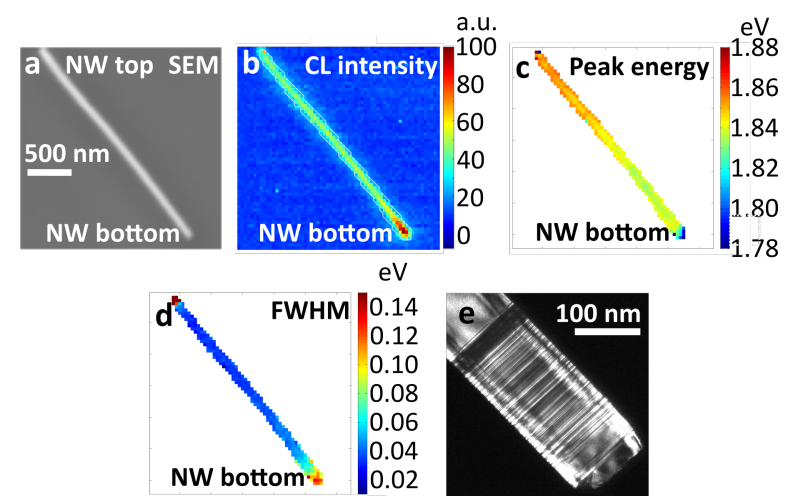
Figure 3

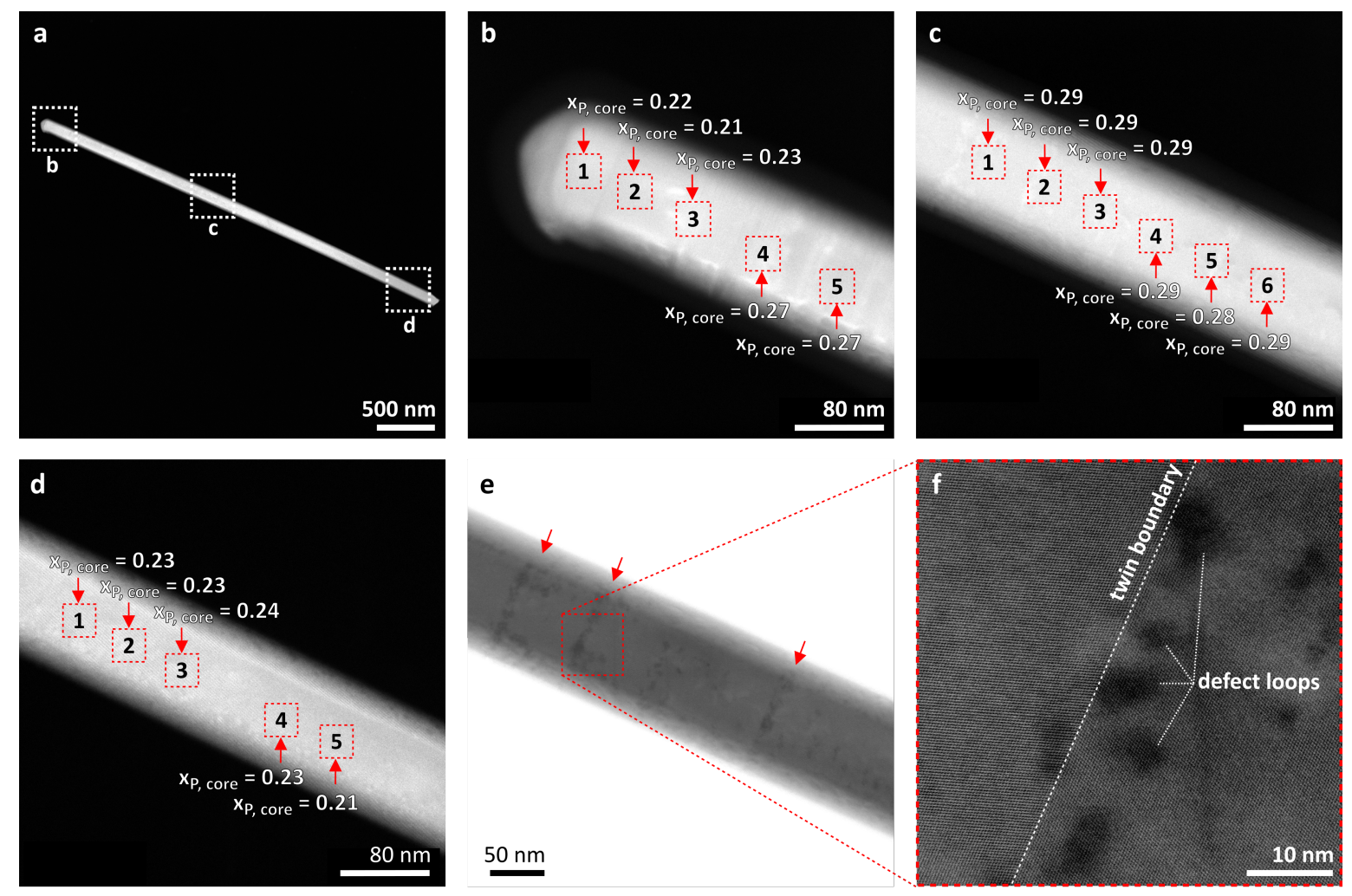


Figure 4
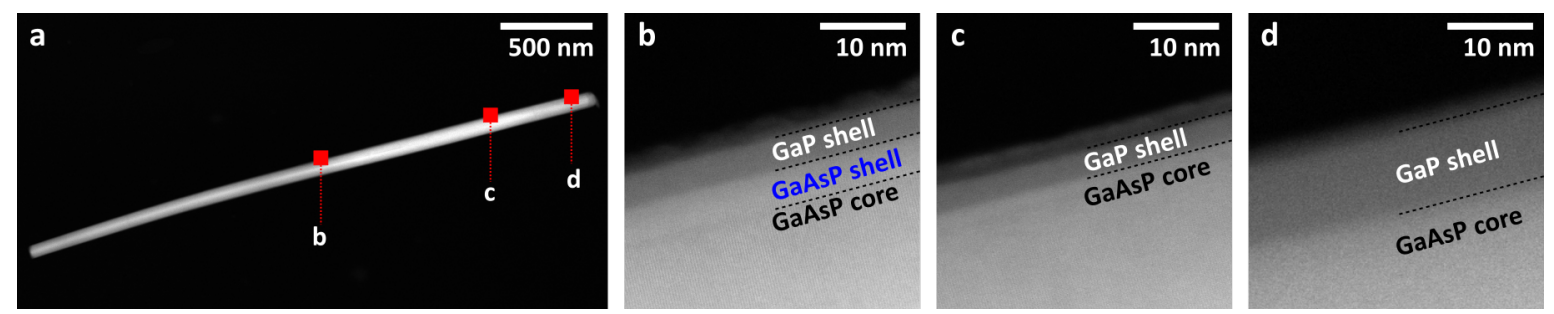


\section{Figure 5}

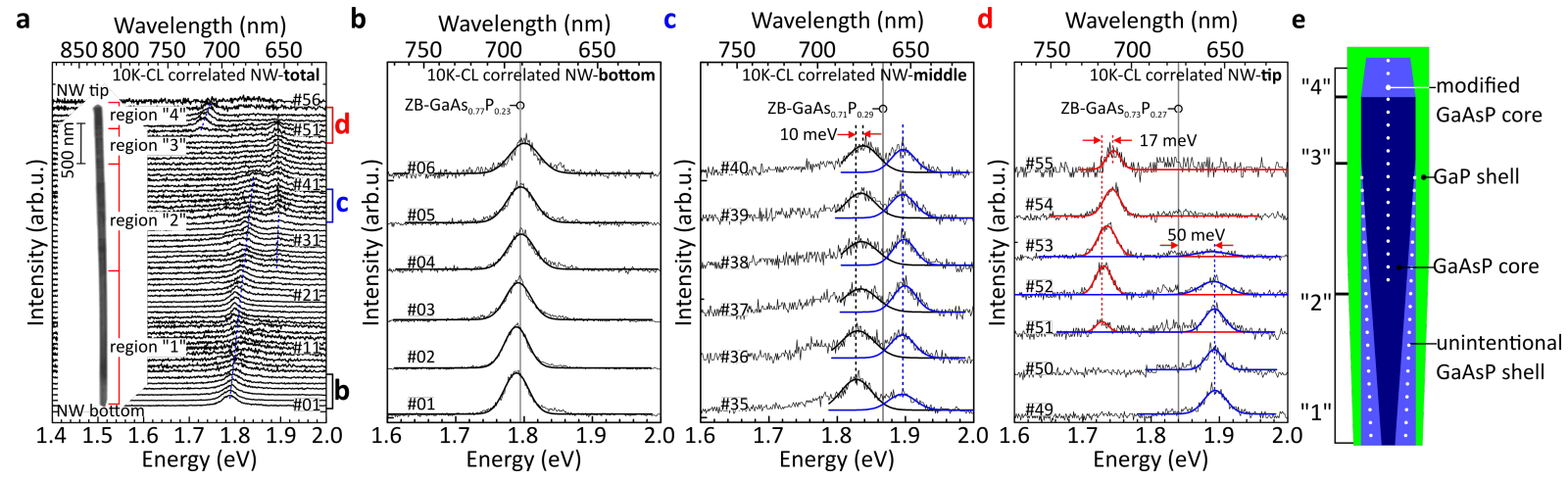


Figure 6

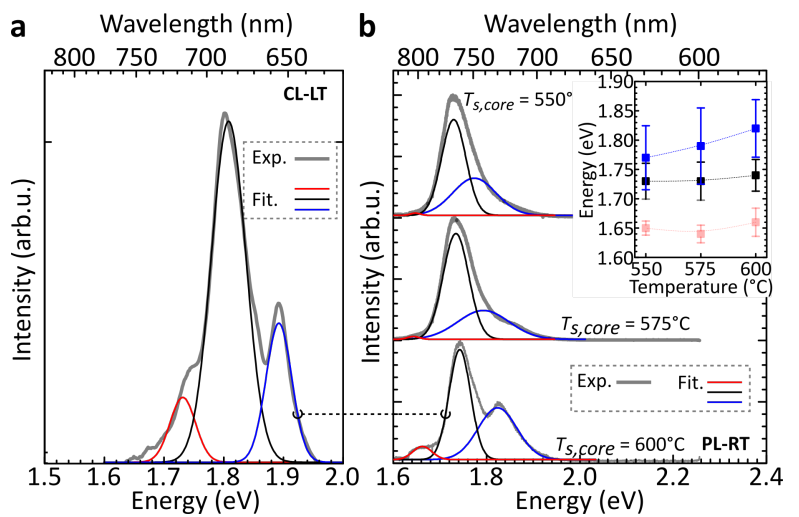

